$4^{\text {th }}$ International Conference on Industrial Engineering and Industrial Management

XIV Congreso de Ingeniería de Organización

Donostia - San Sebastián, September $8^{\text {th }}-10^{\text {th }} 2010$

\title{
Descripción detallada de las Variables de Decisión en Modelos basados en Programación Matemática en un contexto de Planificación Colaborativa de una Red de Suministro/Distribución (RdS/D)**
}

\author{
Detailed description of the Decision Variables in Mathematical \\ Programming Models in a Collaborative Planning Framework of Supply \\ and Distribution Networks (SDN)
}

\author{
David Pérez, Francisco-Cruz Lario y María del Mar Alemany \\ Centro de Investigación en Gestión e Ingeniería de Producción (CIGIP). \\ Universidad Politécnica de Valencia, Calle Camino de Vera s/n. Edificio 8G.Valencia 4607I. \\ dapepe@omp.upv.es fclario@omp.upv.es mareva@omp.upv.es
}

Fecha de recepción: 8-9-2010

Fecha de aceptación: 27-10-2010

Resumen: En este artículo se ha analizado de qué componentes constaría un Modelo determinista basado en Programación Matemática en un contexto de Planificación Colaborativa en Redes de Suministro/Distribución (RdS/D). Aunque sólo se ha considerado la descripción detallada de las Variables de Decisión, la descripción completa de todos los componentes del Modelo ha permitido la definición de una Metodología que tiene como objetivo modelar analíticamente el Proceso de Planificación Colaborativa en cualquier RdS/D, pudiendo ser ésta de diferentes grados de complejidad, tanto desde el punto de vista Físico (Recursos/ĺtems), como desde el punto de vista Organizacional-Decisional e Informacional.

Palabras Clave: planificación colaborativa, modelado, programación matemática, variables de decisión.

Abstract: In this paper is analyzed what components make up a Mathematical Programming Deterministic Model in a Collaborative Planning framework of Supply and Distribution Networks (SDN). Although it is only considered the detailed description of its Decisión Variables, its whole description has allowed a Methodology Definition whose main goal is the analytic modelling of the Collaborative Planning Process in SDN's with different degrees of complexity, either the Physical point of view or the Organizational-Decisional and Informational one.

Keywords: collaborative planning, modelling, mathematical programming, decision variables organizational-decisional and informational one.

\section{Introducción}

En el presente trabajo se analiza de qué partes constaría un Modelo basado en Programación Matemática en un contexto de Planificación Colaborativa en Redes de Suministro/Distribución (RdS/D), distinguiendo entre parte de Definición (Índices, Conjuntos, Variables de Decisión y Parámetros) y de Modelado (Criterio y Campo de Decisión), realizándose sólo una descripción detallada de la primera de ellas, la parte de Definición, y más concretamente del componente Variables de Decisión.

La descripción detallada de la parte de Modelado se puede encontrar en (Pérez y otros, 2009), en la que, de manera análoga, se hace una descripción detallada de sus componentes: Criterio y Campo de Decisión.

Todo lo anterior tendrá como objetivo final proponer una Metodología para el desarrollo de Modelos

\footnotetext{
* Este trabajo se deriva de la participación de sus autores en un proyecto de investigación Feder-Cicyt titulado RdS-2V.RDSINC «De la Planificación a la Ejecución en la Cadena (Red) de Suministro. Dos visiones diferentes y sus herramientas».
} 
Deterministas basados en Programación Matemática para la Planificación Colaborativa de RdS/D (Pérez y otros, 2008). $\bigcirc$ dicho de otra forma, plantear una Metodología que indique los pasos a seguir para desarrollar Modelos Deterministas basados en Programación Matemática en cada uno de los Centros de Decisión/Actividades Decisionales identificadas previamente en el Modelado del Proceso (Pérez, 20 I0), considerando todo tipo de interacciones, jerárquicas o no (Schneeweiss y Zimmer, 2004) que pudieran aparecer en el modelado.

A su vez, la Metodología anterior tendrá como premisas fundamentales:

- el Marco Conceptual descrito tanto en Alarcón y otros (2007) en la que se consideraron las visiones física, organizacional, decisional e informacional, como en Pérez y otros (2008a, 2008b) en la que se abordó la visión más representativa del Proceso de Planificación Colaborativa, esto es la Visión Decisional.

- el Modelo del propio Proceso, obtenido también metodológicamente en Pérez (2010).

En la Figura I se muestra gráficamente todo lo comentado en esta introducción.

\section{Aspectos previos}

El Modelado basado en Programación Matemática se considera estructurado en dos partes: parte de Definición y parte de Modelado (Figura 2).

Tanto la parte de Definición como la parte de Modelado se basan en el Modelo de Referencia Analítico Decisional de un Centro de Decisión genérico $\left(C D^{M}\right)$ desarrollado también metodológicamente en Pérez y otros (2008a).

La parte de Definición equivaldría a la Estructura de Soporte descrita en el Modelo de Referencia Decisional, la cual venía dada por la definición de dos componentes, unas Variables de Decisión $X^{M}$ y una Información de Entrada le ${ }^{M}$ (la cual se identificará mediante una serie de«Parámetros»). Además, como ayuda a la definición de dichos Parámetros y Variables de Decisión, se definieron previamente dos componentes más, los Índices y los Conjuntos.

La parte de Modelado equivaldría a la Estructura Principal descrita en el Modelo de Referencia Decisional. Dicha parte de Modelado estaría compuesta por dos componentes, un Criterio y un Campo de Decisión.

Figura 1

Modelado analítico del Proceso de Planificación Colaborativa en RdS/D

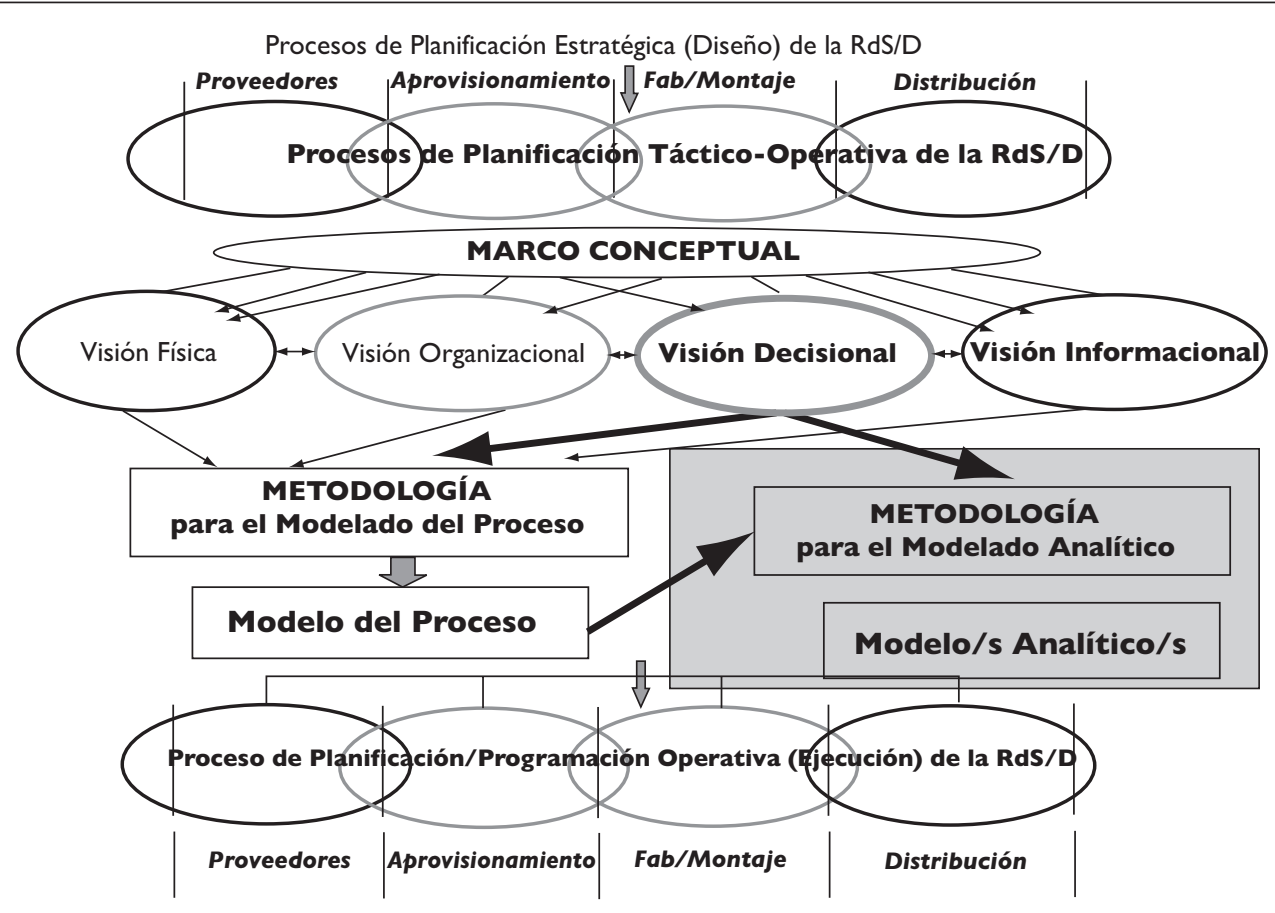

Dirección y Organización, Núm. 42, Diciembre 2010 | ISSN (On line): 2171 -6323 - ISSN (Print): II 32-I75X 
Como ya se ha dicho anteriormente, por razones de espacio, únicamente se considera el componente Variables de Decisión correspondiente a la parte de Definición, detallándose cómo se obtendría para un $C D^{M}$ y cómo sería su representación analítica en un Modelo Determinista basado en Programación Matemática con las características antes señaladas.

Figura 2

Partes y componentes de un Modelo basado en Programación Matemática

\section{PARTE DE MODELADO}

- Criterio

- Campo de Decisión

\section{PARTE DE DEFINICIÓN}

- Índices

- Conjuntos

- Parámetros

- Variables de Decisión

Sea $Z^{M}$ el Modelo Decisional de $C D^{M}$, de manera que $Z^{M}=Z^{M}\left(C^{M}, A^{M}\right)$, donde $C^{M}$ es el Criterio y $A^{M}$ el Campo de Decisión de CDM. La resolución de dicho Modelo Decisional $Z^{M}$ pretende asignar un valor a las Variables de Decisión $\left(\mathrm{X}^{\mathrm{M}}\right)$ que proporcione el mejor valor del Criterio (CM) y cumpla con las limitaciones expresadas en el Campo de Decisión ( $\left.A^{M}\right)$.
En un contexto de Planificación Colaborativa de RdS/D, tanto la definición de las Variables de Decisión como la de los demás componentes, no sólo tendrá en cuenta aspectos ligados al Alcance del propio $C D^{M}$, sino que además se tendrán en cuenta, en mayor o menor medida, aspectos referentes a otros CD ${ }^{M}$ del Entorno Decisional, ya que las decisiones tomadas por unos afectan, restringen o condicionan a las decisiones tomadas por otros. (Figura 3).

\section{Parte de Definición: Variables de Decisión}

Las Variables de Decisión $\left(X_{k}{ }^{M}\right)$ representan una «característica o propiedad desconocida» de un Índice ó Conjunto, cuyo valor será determinado una vez ejecutado el Modelo de Decisión $Z_{k}{ }^{M}$ correspondiente a una Actividad Decisional $k\left(A D_{k}\right)$ de un $C D^{M}$ genérico.

Dichas Variables de Decisión se definirán sobre los Índices y Conjuntos anteriores, de manera que es necesario que estos se hayan definido previamente.

En la Visión Decisional del Marco Conceptual (Pérez y otros, 2007) se definieron ya los distintos tipos de Variables de Decisión de un CD ${ }^{M}$ genérico: Variables Locales $\left(X_{k l}^{M}\right)$ y Variables por Interdependencia, distinguiendo en estas últimas las Variables Globales $\left(X_{k i}{ }^{M}\right)$, que podían ser Finales o no Finales.

\section{ACTIVIDAD DECISIONAL}

¿Quién la realiza?: Responsable del CD (Recurso Humano y/o informático)

¿Cuándo se realiza?: Según Período de Replanificación/Orden de ejecución

¿Qué se realiza?: Plan Operacional Táctico u Operativo (Información de Salida)

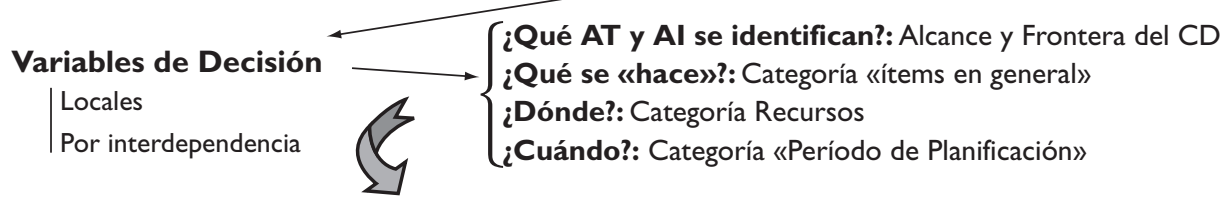

¿Cómo se realiza?:

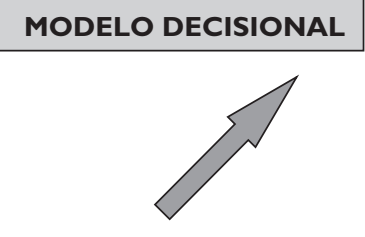

Información de Entrada Local

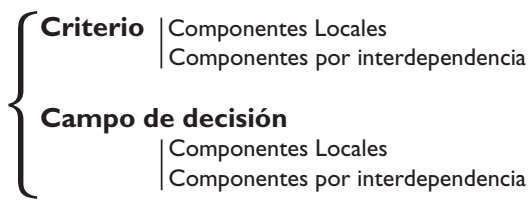

Por Interdependencia

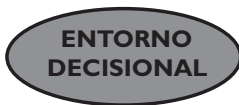




\section{I. Variables de Decisión Locales}

Las Variables de Decisión Locales $\left(X_{k 1}^{M}\right)$ (ó simplemente Variables Locales) dependerán básicamente de cuál es el Alcance/Frontera del $C D^{M}$ en cuestión y su relación con el Entorno, y del Estado de Agregación/Grado de Detalle con el que éste se considera (ambos aspectos cubiertos una vez definidos los Índices y Conjuntos Locales).

¿Cómo se formulan las Variables Locales $\left(X_{k 1}{ }^{M}\right)$ ?

En primer lugar se definirán todas las Variables de Decisión del $C D^{M}$ en cuestión, y en segundo lugar se distinguirá entre aquellas que son Variables Locales y aquellas que son Variables por Interdependencia, como se especificará posteriormente.

Dependiendo de las Interacciones Decisionales de dicho $\mathrm{CD}^{M}$ con los $C D$ pertenecientes a su Entorno, o lo que es lo mismo, del grado de Planificación Colaborativa actual, habrá mayor o menor número de Variables Locales o por Interdependencia entre el conjunto de Variables de Decisión definidas inicialmente.

Dichas Variables de Decisión se formularán en primer lugar sobre cada una de las Actividades de Transformación (AT), esto es, Producción-Operaciones, Almacenamiento y Transporte (pertenecientes al Alcance del $C D^{M}$ ) y sobre cada una de las Actividades de Interconexión (Al), esto es, Compras y Ventas, (pertenecientes a la Frontera del $C D^{M}$ ), sobre las cuales se toman Decisiones específicas (en forma de Plan Operacional) en un Nivel Decisional Táctico y/u Operativo.

Para ello, en el Marco Conceptual se enumeraron las distintas Variables específicas que tenía sentido incluir para la «Toma de Decisiones» en cada una de dichas AT/Al. En este caso, desde el punto de vista analítico, se han expresado de forma abreviada con letras mayúsculas, como se indica a continuación:

En lo que se refiere a las AT:

- Producción-Operaciones: Cantidad a produciroperar (PR), Producir-Operar o no (YPR - YOP), Realizar un Cambio en la Producción o no (YFPR), Capacidad normal (C), Capacidad extra (CEX), Capacidad ociosa (COC)

- Almacenamiento: Cantidad a almacenar/inventariar (IN), Cantidad por debajo del Stock de Seguridad Objetivo (SS-)

- Transporte: Cantidad a transportar (TR), Transportar o no (YTR)

En lo que se refiere a las Al:

- Compras: Cantidad a comprar/subcontratar (CO)

- Ventas: Cantidad a vender (VE), Cantidad a diferir (DIF), Cantidad a rechazar (REC)

En segundo lugar, la Variable Local definida en cualquiera de las AT/AI anteriores tiene sentido en cuánto responde a un «Qué», «Dónde» y «Cuándo» (Categorías «Ítems en General», «Recursos» y «Período de Planificación»), para lo cual se considerarán los Índices definidos previamente en la Parte de Definición. Dichos Índices se colocarán como subíndices separados por comas.

En la Tabla I se muestra tabularmente cómo se formularía una Variable de Decisión Local:

Tabla I

Definición tabular de las Variables de Decisión Locales

\begin{tabular}{|c|c|c|c|}
\hline ALCANCE/FRONTERA & \multicolumn{3}{|c|}{ Categorías/Tipos de Elementos (Índices Básicos) } \\
\hline AT/AI - Var. específica & Ítems en General & Recursos & Período de Planificación \\
\hline Compras & \multirow{5}{*}{$\begin{array}{c}\text { Ítem } \\
\text { Grupo de Ítems }\end{array}$} & \multirow{5}{*}{$\begin{array}{c}\text { Alternativa/Línea } \\
\text { Etapa (intra-Nodo) } \\
\text { Nodo } \\
\text { Puntos de Venta } \\
\text { Grupo de Nodos } \\
\text { Grupos de Puntos de Venta }\end{array}$} & \multirow{5}{*}{$\begin{array}{c}\text { Diario } \\
\text { Semanal } \\
\text { Bimensual } \\
\text { Trimestral } \\
\text { Cuatrimestral } \\
\text { Semestral } \\
\text { Anual }\end{array}$} \\
\hline Producción-Operaciones & & & \\
\hline Almacenamiento & & & \\
\hline Transporte & & & \\
\hline Ventas & & & \\
\hline
\end{tabular}


- Una Variable específica definida sobre alguna de las AT/AI anteriores.

- UnTipo de Elemento (Indice) perteneciente, en principio, a cada una de las tres Categorías, sin los cuales no tendría sentido la Variable específica anterior.

Por ejemplo se podría definir la Variable de Decisión

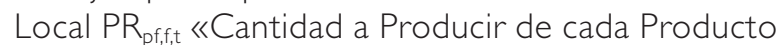
Final (Índice de la Categoría Ítems en General) en cada Nodo de la Etapa de Fabricación-Montaje (Índice de la Categoría Recursos) en cada Mes (Índice de la Categoría Período de Planificación)».

Por último, conviene tener presente las siguientes reglas:

- Una Variable siempre se definirá sobre uno o más Índices, ya que la Categoría «Período de Planificación» siempre existirá, tal y como se indicó en la Visión Decisional.

- Una Variable sólo se definirá mediante Índices que por defecto corresponderán a Conjuntos Locales Básicos. En caso contrario, esos Índices formarán Conjuntos Relacionales (o por Interdependencia), los cuales se deben tener en cuenta a efectos de modelado y posterior resolución.

- Ejemplo: se puede definir la Variable PR i,l,n,t: «cantidad a producir de cada Ítem en cada Línea de cada Nodo en cada Mes»». En este caso se han definido cuatro Índices, entre los cuales, por defecto, se supone que corresponden a Conjuntos Básicos, $i € I, I € L, n \in N$ y $t € T$, por lo que existirían I* $L^{*} \mathrm{~N} * T$ posibles valores para dicha Variable. En caso que no fuera así, sería recomendable a efectos de modelado y posterior resolución, que se acotasen los posible valores, sin más que definir, en caso que así sea, Conjuntos Relacionales (o por Interdependencia), como pudiera ser $i € \mid(I)$ y $\mid € L(n)$.

Una vez definidas todas las Variables de Decisión, sólo se comportarán como Variables Locales aquellas que:

- no «conectan» con Variables Globales enviadas a modo de Instrucción desde $C D^{\top}$

- no se «envian» a modo de Instrucción (en forma de Variables Globales) a $C D^{B}$

De lo anterior deriva que el resto se considerarán Variables por Interdependencias. No obstante, ade- más de las anteriores existen otro tipo de Variables por Interdependencia que no se han especificado hasta ahora, tal y como se detalla a continuación.

\subsection{Variables de Decisión por Interdependencia}

Las Variables de Decisión por Interdependencia (o simplemente Variables por Interdependencia) corresponderán a aquellas que «interaccionen» en mayor o menor medida con el Entorno del CD ${ }^{M}$ en cuestión.

Además, al igual que las Variables Locales, su formulación tendrá en cuenta el Estado de Agregación/Grado de Detalle con el que dicho Entorno es considerado, todo ello cubierto a partir de la parte de Definición con los Índices y Conjuntos por Interdependencia.

Según el Marco Conceptual y la Metodología para el Modelado del Proceso mencionadas en el apartado de introducción, un $C D^{\top}$, con carácter general, activará/ejecutará su Actividad Decisional $k\left(A D_{k}\right)$ representada mediante su Modelo de Decisión $Z_{k}{ }^{\top}$ (con menor o mayor grado de Anticipación de $C D^{M}$ ) y obtendrá unos valores concretos para sus Variables Decisión $\left(X_{k}^{\top}\right)$, de las cuales unas se tratarán de Variables Locales $\left(X_{k}{ }^{\top}\right)$ y otras de Variables por Interdependencia $\left(\mathrm{X}_{\mathrm{ik}}{ }^{\top}\right)$.

Algunas de las Variables por Interdependencia (ya sea directamente o mediante una Función de las mismas) se convertirán en Variables Globales, tanto Finales como No-Finales $\left(\mathrm{XF}_{\mathrm{i} k}{ }^{\mathrm{TM}} ; \mathrm{XNF}_{\mathrm{i} k}{ }^{\mathrm{TM}}\right)$ para $\mathrm{CD}^{\mathrm{M}}$ por medio de una Instrucción (IN).

Dichas Variables Globales enviadas mediante una IN desde $C D^{\top}$ «conectarán» con las Variables por Interdependencia de $C D^{M}$ con respecto de $C D^{\top}$ $\left(\mathrm{X}_{\mathrm{i}}{ }^{\mathrm{MT}}\right)$, las cuales podrán variar o no en función de si las Variables Globales anteriores eran Finales o no Finales.

Además $C D^{M}$, teniendo en cuenta las anteriores $\mathrm{Va}$ riables por Interdependencia con $C D^{\top}$, deberá distinguir entre el resto de sus Variables de Decisión cuáles son Locales y cuáles son de Interdependencia con respecto a $C D^{B}$, es decir, cuáles de estas últimas (ya sea directamente o mediante una Función de las mismas), una vez ejecutado el Modelo Decisional $Z_{k}{ }^{M}$, con menor o mayor anticipación de $C D^{B}$, se convertirán en Variables Globales, tanto Finales como No-Finales $\left(\mathrm{XF}_{\mathrm{ik}}{ }^{\mathrm{MB}} ; \mathrm{XNF}_{\mathrm{ik}}{ }^{\mathrm{MB}}\right)$ que serán enviadas a $C D^{B}$ por medio de una Instrucción (IN). 
Todo lo anterior indica que, como se ha dicho al final del apartado anterior, además de las Variables por Interdependencia definidas hasta ahora, es decir, aquellas que «conectan» con Variables Globales enviadas a modo de Instrucción desde $C D^{\top}$ y aquellas que se «envian» a modo de Instrucción (en forma de Variables Globales) a $C D^{B}$, se deban de definir dos tipos más:

- las que se derivan de las desviaciones (anticipadas o no) sobre las Variables por Interdependencia (Globales) definidas inicialmente.

- las que se definen como consecuencia de la anticipación de Variables Locales de $C^{B}$ (o en su caso, de $C D^{\top}$ ).

A continuación se realiza una clasificación de las Variables por Interdependencia $\left(X_{i k}{ }^{M}\right)$ dependiendo de la interacción, en su caso, de $C D^{M}$ con los CD de su Entorno (Figuras 4 y 5 ):

- Para cada CD ${ }^{T t}:\left(\mathrm{X}_{\mathrm{ki}}^{\mathrm{MTt}}\right.$ ó simplemente $\left.\mathrm{X}_{i}^{\mathrm{MTt}}\right)$

- Instrucciones enviadas desde CD ${ }^{T t}$

* X ${ }^{\text {MTt: }}$ Variables de Decisión de CDM que «conectan» con las Variables Globales «enviadas» (a modo de IN), desde cada CD ${ }^{T t}$

* $\mathrm{X}^{+\mathrm{TtM}}, \mathrm{X}^{-\mathrm{TtM}}$ : desviaciones positivas o negativas sobre las Variables Globales «enviadas» (a modo de IN) desde CD ${ }^{T t}$

- Anticipaciones de la Reacción de CDM

* XMTt: Variables de Decisión que se envían (a modo de $R$ ) a $C D^{T t}$

* ant_ $X^{+M T t}$; ant_ $X^{-M T t}$ : desviaciones positivas o negativas anticipadas sobre las Variables Globales «enviadas» (a modo de R) desde CDM

* ant_X $X^{T t}$ :Variables Locales de CD ${ }^{T t}$ que anticipa CD ${ }^{M}$

- Para cada CD ${ }^{\mathrm{Te}}:\left(\mathrm{X}_{\mathrm{ki}}^{\mathrm{MTe}}\right)$

- Instrucciones enviadas desde CDTe

* XMTe:Variables de Decisión de CDM que «conectan» con las Variables Globales «enviadas» (a modo de IN), desde cada CD ${ }^{\text {Te }}$

* $\mathrm{X}^{+\mathrm{TeM}} ; \mathrm{X}^{\text {-TeM}}$ : desviaciones positivas o negativas sobre las Variables Globales «enviadas» (a modo de IN) desde CD ${ }^{\text {Te }}$
- Anticipaciones de la Reacción de CDM

* XMTe: Variables de Decisión que se envían (a modo de R) a $C D^{\text {Te }}$

* ant_X+MTe; ant_X-MTe: desviaciones positivas O negativas anticipadas sobre las Variables Globales «enviadas» (a modo de R) desde CD ${ }^{M}$

* ant_X $\mathrm{X}^{\mathrm{Te}}$ Variables Locales de CD ${ }^{\mathrm{Te}}$ que anticipa $C D^{M}$

- Para cada CD ${ }^{\mathrm{Bt}}:\left(\mathrm{X}_{\mathrm{ki}}^{\mathrm{MBt}}\right)$

- Anticipaciones a la Instrucción de CDM

* XMBt: Variables de Decisión Globales que $C D^{M}$ envía (a modo de IN) a CD ${ }^{B t}$

* ant_X ${ }^{+M B t}$; ant_X-MBt: desviaciones positivas O negativas anticipadas sobre las Variables Globales «enviadas» (a modo de IN) desde CDM

* ant_ $X^{B t}$ :Variables Locales de CD ${ }^{\text {Bt }}$ que anticipa CD ${ }^{M}$

- Reacciones desde $C D^{B t}$

* XMBt:Variables de Decisión de CDM que «conectan» con las Variables Globales «enviadas» (a modo de R), desde cada $C D^{\text {Bt }}$

* $\mathrm{X}^{+\mathrm{BtM}} ; \mathrm{X}^{-\mathrm{BtM}}$ : desviaciones positivas o negativas sobre las Variables Globales «enviadas» (a modo de R) desde CD ${ }^{T t}$

- Para cada CD ${ }^{B e}:\left(X_{k i}{ }^{M B e}\right)$

- Anticipaciones a la Instrucción de CDM

* XMBe: Variables de Decisión Globales que $C D^{M}$ envía (a modo de IN) a CD ${ }^{B t}$

* ant_X+MBe; ant_X-MBe: desviaciones positivas O negativas anticipadas sobre las Variables Globales «enviadas» (a modo de IN) desde CD ${ }^{M}$

* ant_X $\mathrm{X}^{\mathrm{Te}}$ :Variables Locales de $\mathrm{CD}^{\mathrm{Be}}$ que anticipa $C D^{M}$

- Reacciones desde CD ${ }^{\text {Be }}$

* XMBe:Variables de Decisión de CDM que «conectan» con las Variables Globales «enviadas» (a modo de R), desde cada CD ${ }^{\mathrm{Be}}$

* $\mathrm{X}^{+\mathrm{BeM}} ; \mathrm{X}^{-\mathrm{Be} M}$ : desviaciones positivas o negativas sobre las Variables Globales «enviadas» (a modo de R) desde CD ${ }^{\mathrm{Te}}$. 
Figura 4

Variables de Decisión Locales y por Interdependencia (I) de un $\mathrm{CD}^{\mathrm{M}}$ genérico

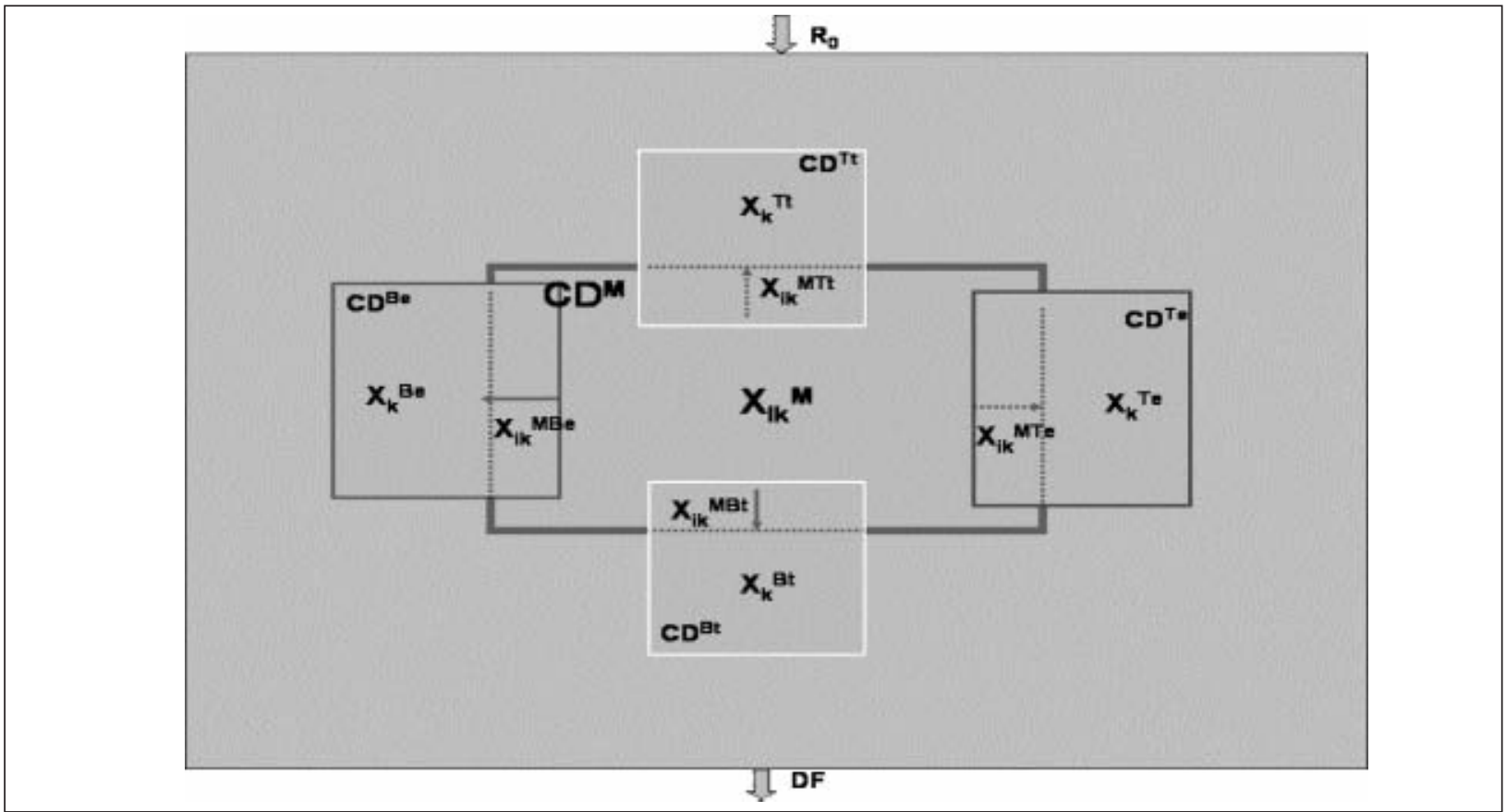

Figura 5

Variables de Decisión Locales y por Interdependencia (II) de un CDM genérico

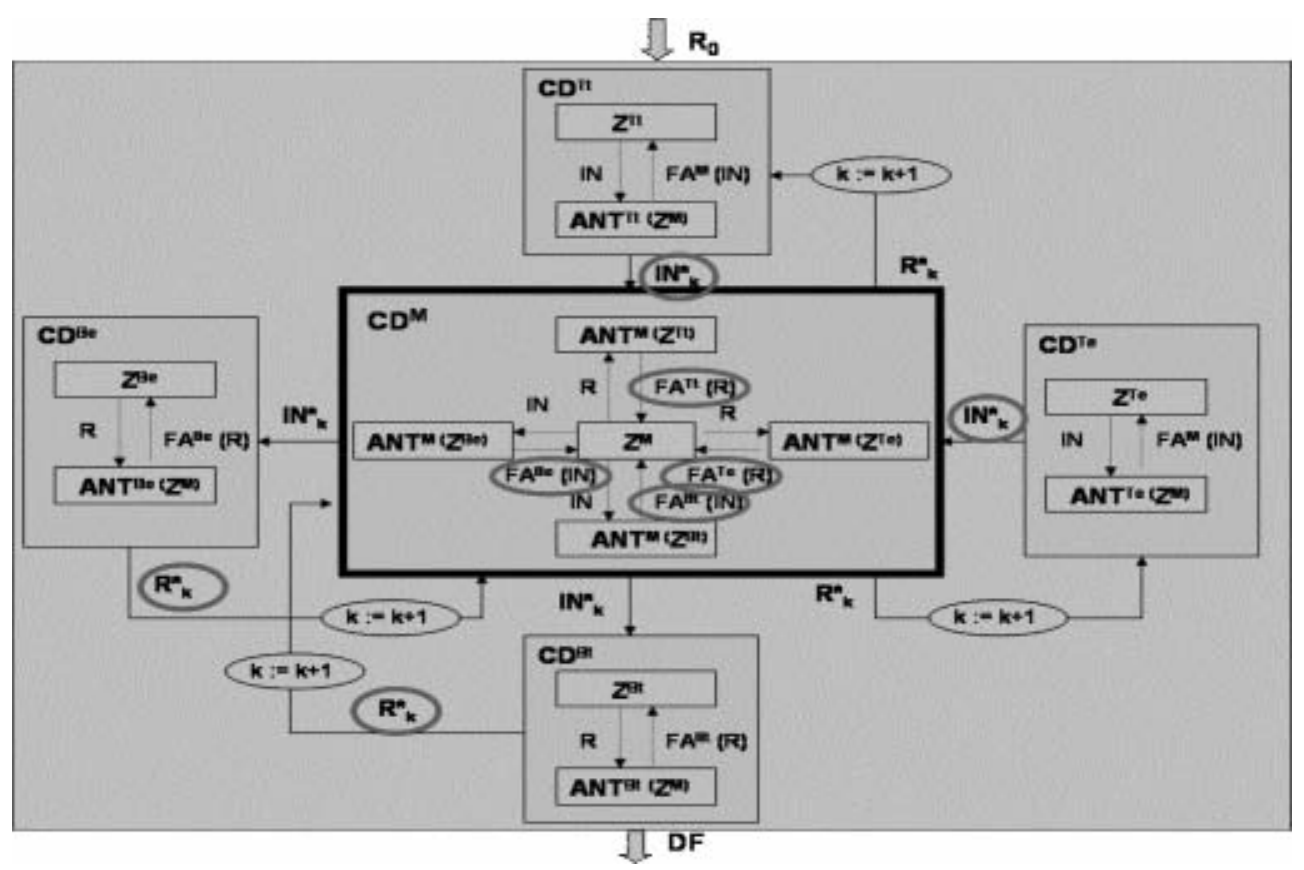

¿Cómo se formulan las Variables por Interdependencia $\left(X_{k i}{ }^{M}\right)$ ?

Se formulan de la misma forma que las Variables Locales, es decir:
- Una Variable específica definida sobre alguna de las AT/Al.

- UnTipo de Elemento (Indice) perteneciente, en principio a cada una de las tres Categorías, sin los 
cuales no tendría sentido la Variable específica anterior. En este caso tendrán especial relevancia los Índices por Interdependencia, también definidos previamente en la Parte de Definición del Modelo.

Por ejemplo, CDM podría definir como Variable por Interdependencia $V E_{p f, d, t}$, si ésta «conectara» con una Variable Global, enviada mediante una Instrucción desde un $C D^{\text {Te }}$, quizá un Plan de Requerimientos/Demanda. En caso contrario, VE $E_{\mathrm{pfd}, \mathrm{d}, \mathrm{t}}$ se comportaría como una Variable Local, probablemente definida a partir de una Previsión de Ventas.

En cuanto a las Variables por Interdependencia debidas a desviaciones se representarán de forma abreviada con letras mayúsculas con un superíndice + o - dependiendo del Tipo de Desviación respecto de la Variable específica original.

- Por Ejemplo $\mathrm{PR}^{+}-\mathrm{OP}^{+}$: Desviación positiva sobre la «cantidad a producir-operar» objetivo

Por último, las Variables por Interdependencia debidas a posibles anticipaciones se representarán mediante el sufijo «ant_», como por ejemplo, ant_PR podría tratarse de una Variable que tratara de anticipar la «cantidad a producir en un Proveedor en cada período».

\section{Conclusiones}

En este artículo se ha analizado de qué partes constaría un Modelo determinista basado en Programación Matemática, distinguiendo entre parte de Definición (Índices, Conjuntos, Variables de Decisión y Parámetros) y de Modelado (Criterio y Campo de Decisión), realizándose sólo una descripción detallada de la primera de ellas, y más concretamente del componente Variables de Decisión, analizando cómo se obtendrían para un $C D^{M}$ genérico y cuál sería su representación analítica.

La descripción detallada anterior, así como de los demás componentes ha sido necesaria para la definición de una Metodología que sirva de ayuda al desarrollo de Modelos Deterministas basados en Programación Matemática para la Planificación Colaborativa de Redes de Suministro/Distribución. Dicha Metodología, que no figura en el presente trabajo, se puede encontrar en (Pérez, 2010) y se compone básicamente de dos bloques:

- Un primer bloque que se basa directamente en la descripción detallada anterior de todos las Partes/
Componentes, y en el que se indica de manera clara y ordenada los pasos que debería seguir cualquier CDM genérico para definir un Modelo Determinista basado en Programación Matemática en el contexto colaborativo antes señalado.

- Un segundo bloque en el que se indican los pasos para el Modelado Analítico general del Proceso de Planificación Colaborativa de RdS/D, haciendo especial énfasis en cómo «interaccionan» los Modelos individuales anteriormente descritos.

Es importante mencionar que la citada Metodología permitirá modelar analíticamente el Proceso de Planificación Colaborativa en cualquier RdS/D, pudiendo ser ésta de diferentes grados de complejidad, tanto desde el punto de vista Físico (Recursos/Ítems), como desde el punto de vista Organizacional-Decisional e Informacional.

El párrafo anterior incide en la importancia y relevancia de la definición previa de un Marco Conceptual y una Metodología para el Modelado del Proceso, tal y como se comentó en el apartado de introducción. Además, no sólo se podrá modelar la situación actual (AS-IS), sino que la Metodología descrita permitirá evaluar diferentes configuraciones o situaciones futuras (TO-BE), a partir de cambios tanto desde el punto de vista Físico, Organizacional-Decisional e Informacional.

En Pérez (20 I0) se realiza, además, una aplicación de la citada Metodología en una RdS/D del Sector de Pavimentos y Revestimientos Cerámicos.

\section{Referencias}

ALARCÓN F.; LARIO F.C.; BOZA A. y PÉREZ D. (2007) «Propuesta de Marco Conceptual para el modelado del proceso de Planificación Colaborativa de Operaciones en contextos de Redes de Suministro/Distribución» Internacional Conference on Industrial Engineering and Industrial Management, Madrid. Vol I, pp 873-882.

ALEMANY M.M.; PÉREZ D.; ALARCÓN F. y BOZA A. (2007). «Planificación Colaborativa en RdS/D mediante Programación Matemática en Entornos Distribuidos» XI Cong. Ingeniería Organización, Madrid.Vol I, pp 853-862.

PÉREZ D.; ALEMANY M.M.; VICENS E. y LARIO F.C. (2007). «Propuesta de Marco Conceptual para el Modelado de la Visión Decisional del proceso de Planificación Colaborativa de una RdS/D» XI Cong. Ingeniería Organización, Madrid.Vol I, pp 893-902.

PÉREZ D.; LARIO F.C. y ALEMANY M.M. (2008a). «Metodología para el modelado Analítico Decisional de un Cen- 
tro de Decisión genérico en un contexto jerárquico de Planificación Colaborativa de una Red de Suministro/Distribución (RdS/D)» II Internacional Conference on Industrial Engineering and Industrial Management, Burgos. Vol I, pp 407-4I6

PÉREZ D.; LARIO F.C. y ALEMANY M.M. (2008b). «Metodología para el desarrollo de Modelos basados en Programación Matemática en un contexto jerárquico de Planificación Colaborativa de una Red de Suministro/Distribución (RdS/D)» II Internacional Conference on Industrial Engineering and Industrial Management, Burgos. Vol I, pp |5|3-1522

PÉREZ D.; LARIO F.C. y ALEMANY M.M. (2009). «Descripción detallada del Criterio y del Campo de Decisión en Modelos basados en Programación Matemática en un contexto jerárquico de Planificación Colaborativa de una Red de Suministro/Distribución (RdS/D)» III Internacional Conference on Industrial Engineering and Industrial Management. Barcelona-Terrassa. Vol I, pp |478-| 488.

PÉREZ, D. (20 I 0). «Desarrollo de un Marco Conceptual y una Metodología para el desarrollo de Modelos Deterministas basados en Programación Matemática para la Planificación Colaborativa de Redes de Suministro / Distribución. Aplicación a Empresas del Sector de Pavimentos y Revestimientos Cerámicos» Tesis Doctoral pendiente de publicación/ Cap.6.

SCHNEEWEISS, CH. y Zimmer K. (2004). «Hierarchical coordination mechanism within the Supply Chain» European Journal of Operational Research. Vol. I53, pp. 687-703. 